\title{
Tunelizador metálico rígido na confecção de fístulas arteriovenosas dos membros superiores
}

\author{
Rigid metallic tunneler for the creation of arteriovenous fistulas in the upper extremity \\ Heraldo Antonio Barbato', Patrick Bastos Metzger², Fernanda Maria Resegue Angelieri², Bruno Lourenção Almeida², \\ Eduardo da Silva Jordão², Fabio Henrique Rossi', Bruno Utiyama da Silva3, Nilo Mitsuru Izukawa ${ }^{4}$
}

\begin{abstract}
Resumo
A qualidade de um acesso vascular condiciona os resultados clínicos dos pacientes tratados por hemodiálise. A criação de um túnel para a acomodação do conduto é uma etapa importante durante a realização do acesso. Desenvolveu-se um tunelizador metálico rígido, empregado na confecção de acessos vasculares. O aparato utilizado apresentou vantagens e segurança nas cirurgias de confecções de acessos venosos para hemodiálise dos membros superiores.
\end{abstract}

Palavras-chave: Insuficiência renal crônica; fístula arteriovenosa; extremidade superior.

\begin{abstract}
The quality of a vascular access determines clinical outcomes of patients on hemodialysis. The creation of the tunnel for conduit insertion is an important step during the access. A rigid metallic tunneler was developed and employed to create arteriovenous fistulas. The device has proven advantageous and safe in operations for the creation of venous accesses for hemodialysis in the upper extremity.
\end{abstract}

Keywords: renal insufficiency, chronic; arteriovenous fistula; upper extremity.

\section{Introdução}

A qualidade de um acesso vascular condiciona os resultados clínicos dos pacientes tratados por hemodiálises periódicas. As complicações originadas dos acessos vasculares causam a maior parte da morbimortalidade desses pacientes e contribuem para a perda do acesso e o aumento do custo nos tratamentos dialíticos ${ }^{1}$.

Atualmente, com a grande manipulação dos pacientes dialíticos, torna-se cada vez menos possível a realização de fistulas arteriovenosas (FAV) distais nos membros superiores (MMSS), seja por falta de um conduto venoso adequado ou por um fluxo arterial diminuído. Por isso, o uso da transposição da veia basílica e a realização de enxertos com próteses sintéticas - politetrafluoroetileno expandido (PTFE) - são uma opção aos pacientes que já não têm condições de realizar acessos vasculares (AV) convencionais.

A cirurgia para confecção de AV com próteses e as transposições venosas nos MMSS envolvem a criação de um túnel para a acomodação do enxerto vascular. Seu trajeto e extensão dependem da posição do segmento arterial escolhido e da extensão do trajeto venoso a ser transposto. A realização de incisões escalonadas para a passagem do conduto aumenta a manipulação dos tecidos e tempo cirúrgico do procedimento, podendo predispor a infecções relacionadas aos AV - complicação grave nos pacientes em processo de hemodiálise².

Trabalho realizado no Instituto Dante Pazzanese de Cardiologia, São Paulo (SP), Brasil.

${ }^{1}$ Cirurgião Vascular da Seção Médica de Cirurgia Vascular do Instituto Dante Pazzanese de Cardiologia, São Paulo (SP), Brasil.

${ }^{2}$ Médicos Residentes da Seção Médica de Cirurgia Vascular do Instituto Dante Pazzanese de Cardiologia, São Paulo (SP), Brasil.

${ }^{3}$ Engenheiro da Seção de Bioengenharia da Fundação Adib Jatene - Instituto Dante Pazzanese de Cardiologia, São Paulo (SP), Brasil.

${ }^{4}$ Chefe da Seção Médica de Cirurgia Vascular do Instituto Dante Pazzanese de Cardiologia, São Paulo (SP), Brasil.

Não foram declarados conflitos de interesse associados à publicação deste artigo.

Submetido em: 21.11.10. Aceito em: 31.05 .11

J Vasc Bras. 2011;10(2):154-159. 


\section{Objetivo}

Avaliar a utilização de aparato tunelizador metálico rígido na confecção de acessos venosos para hemodiálise nos MMSS.

\section{Métodos}

O aparato tunelizador metálico rígido foi desenvolvido no Laboratório de Bioengenharia do Instituto Dante Pazzanese de Cardiologia (Figura 1) e consiste de uma estrutura cilíndrica

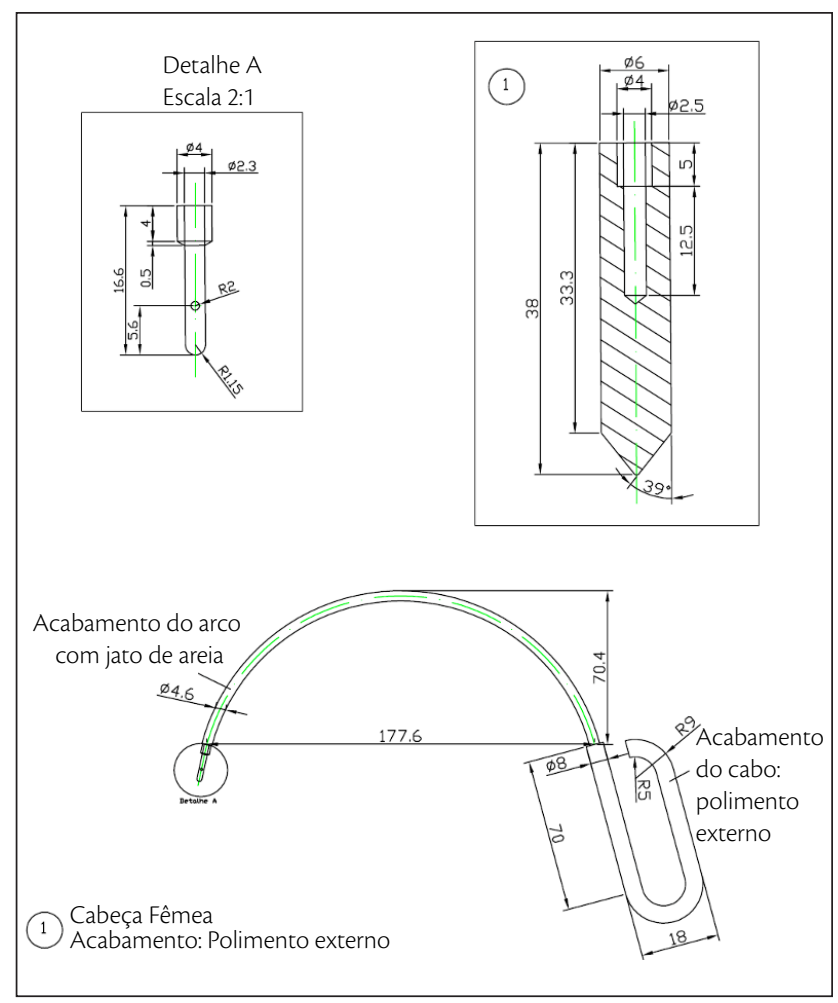

Figura 1 - Tunelizador metálico rígido e curvo para realização de fístulas arteriovenosas dos membros superiores - projeto industrial. Dimensões do corpo e manopla do tunelizador. Detalhe de sua ponta externa cilindrica côncava ajustável à ponta interna do tunelizador. R: representação da medida; valores de comprimento em $\mathrm{mm}$.

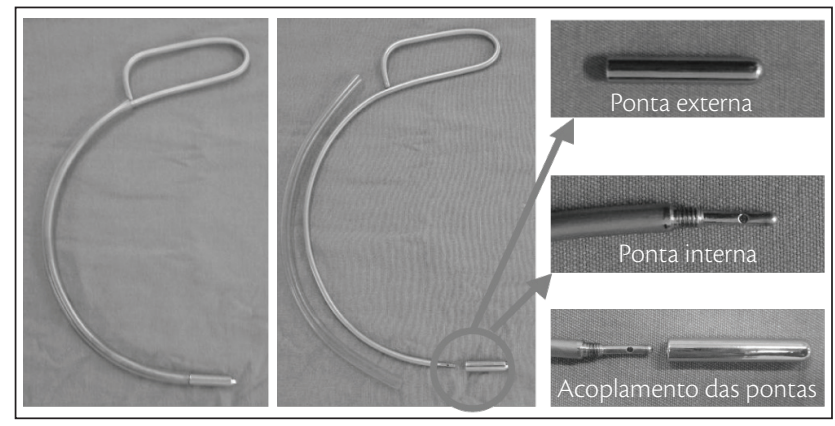

Figura 2 - Tunelizador metálico rígido curvo. Detalhe de ponta interna (tunelização de conduto venoso) e ponta externa (tunelização de prótese de $6 \mathrm{~mm}$ ). rígida e côncava composta de: 1) fio de aço inoxidável rígido interno, com manopla em extremidade proximal e ponta cônica intercambiável em extremidade distal; 2) bainha cilíndrica externa de polietileno transparente (Figura 2).

\section{Desenho do estudo}

Trata-se de um estudo prospectivo, não randomizado, realizado em um único centro, durante o período de agosto de 2010 a novembro de 2011. Admitiram-se ao presente estudo 11 pacientes com insuficiência renal crônica dialítica sem condições de realização de FAV distais (fístulas radiocefálicas, braquiocefálicas ou braquiobasílicas), seja pela ausência de conduto venoso ideal ou pela falha de FAV autólogas prévias.

Os pacientes foram acompanhados durante o perioperatório, no retorno ambulatorial de 7 e de 30 dias. Analisaram-se: 1) o sucesso terapêutico inicial, com a presença de frêmito no conduto venoso ou prótese sintética após a cirurgia; 2) a presença de complicações relacionadas ao procedimento no $7^{\circ}$ e $30^{\circ}$ dia pós- operatório (PO); 3) dificuldade de utilização da FAV durante a hemodiálise, seja por dificuldade de punção ou baixo fluxo na hemodiálise, aqui definida como velocidade de diálise $<250 \mathrm{~mL} / \mathrm{min}$.

O aparato metálico foi utilizado em todas as cirurgias de confecção de acessos vasculares do membro superior realizadas, independentemente do tipo, trajeto e extensão escolhidos.

\section{Resultados}

A experiência inicial obtida com o aparato idealizado foi realizada em 11 pacientes com insuficiência renal crônica (IRC) dialítica: em 6 confeccionaram-se fístulas axilobraquiais com PTFE de $6 \mathrm{~mm}$; em 3, realizaram-se transposições de veia basílica e, em 2, realizaram-se fístulas

Tabela 1 - Dados demográficos e fatores de risco $(n=11)$

\begin{tabular}{lc}
\hline Características da população & Valor \\
\hline Idade média & $47,8(26-68$ anos) \\
Sexo $(M / F)$ & $6 / 5(55 / 45 \%)$ \\
Raça (B/P/N) & $5 / 4 / 2(45 / 36 / 19 \%)$ \\
Hipertensão & $11(100 \%)$ \\
Diabetes mellitus & $5(45 \%)$ \\
Infarto do miocárdio prévio & $2(18 \%)$ \\
Revascularização do miocárdio & $1(9 \%)$ \\
Tabagismo (ativo ou abstêmico há 1 ano) & $4(36 \%)$ \\
No de cateteres prévios >2 (veia jugular e/ou subclávia) & $8(73 \%)$ \\
por paciente & \\
№ de fístulas prévias em MMSS >2 por paciente & $10(91 \%)$ \\
\hline
\end{tabular}

$\mathrm{O}$ valor expressa o número de pacientes. M: masculino; F: feminino. B: branca; $\mathrm{P}$ : parda; $\mathrm{N}$ : negra. MMSS: membros superiores. 
radiobasílicas com PTFE de $6 \mathrm{~mm}$ de diâmetro. As características e comorbidades da população estudada estão listadas nas Tabelas 1 e 2.

Suas características de rigidez e curvatura permitiram a tunelização eficiente do conduto em todos os casos,

Tabela 2 - Grupo de pacientes operados com o tunelizador metálico rígido

\begin{tabular}{|c|c|c|c|c|}
\hline Paciente & Idade & Sexo & Indicação & Cirurgia \\
\hline GG & 62 & $\mathrm{~F}$ & IRC dialítica & $\begin{array}{l}\text { FAV axilobraquial } \\
\text { com PTFE de } 6 \mathrm{~mm}\end{array}$ \\
\hline SMMS & 47 & $\mathrm{~F}$ & IRC dialítica & $\begin{array}{l}\text { FAV axilobraquial } \\
\text { com PTFE de } 6 \mathrm{~mm}\end{array}$ \\
\hline MGGC & 53 & M & IRC dialítica & $\begin{array}{l}\text { FAV axilobraquial } \\
\text { com PTFE de } 6 \mathrm{~mm}\end{array}$ \\
\hline TBS & 39 & M & IRC dialítica & Transposição de veia basílica \\
\hline RVS & 26 & $\mathrm{~F}$ & IRC dialítica & Transposição de veia basílica \\
\hline EJS & 42 & $\mathrm{~F}$ & IRC dialítica & $\begin{array}{l}\text { FAV radiobasílica } \\
\text { com PTFE de } 6 \mathrm{~mm}\end{array}$ \\
\hline SJS & 68 & M & IRC dialítica & $\begin{array}{l}\text { FAV axilobraquial } \\
\text { com PTFE de } 6 \mathrm{~mm}\end{array}$ \\
\hline $\mathrm{BOL}$ & 57 & M & IRC dialítica & $\begin{array}{l}\text { FAV axilobBraquial } \\
\text { com PTFE de } 6 \mathrm{~mm}\end{array}$ \\
\hline ES) & 31 & M & IRC dialítica & Transposição de veia basílica \\
\hline MJA & 56 & M & IRC dialítica & $\begin{array}{l}\text { FAV radiobasílica } \\
\text { com PTFE de } 6 \mathrm{~mm}\end{array}$ \\
\hline FMR & 45 & $\mathrm{~F}$ & IRC dialítica & $\begin{array}{l}\text { FAV axilobraquial } \\
\text { com PTFE de } 6 \mathrm{~mm}\end{array}$ \\
\hline
\end{tabular}

M: masculino; F: feminino; FAV: fístula arteriovenosa; IRC: insuficiência renal crônica; PTFE:politetrafluoroetileno expandido. propiciando uma boa distância para o sítio de punção e sem lesões de estruturas vizinhas. Não foram observadas quaisquer complicações, como sangramentos, hematomas com necessidade de intervenção cirúrgica, trombose, lesões neurológicas, insuficiência arterial no membro acometido e infecções no período de 30 dias de acompanhamento (Tabela 3). A ponta cônica distal com duplo diâmetro, intercambiável, permitiu que o mesmo aparato pudesse ser utilizado na cirurgia de confecção de acessos venosos de MMSS, com uso de conduto venoso ou prótese de PTFE de $6 \mathrm{~mm}$, no nível do braço ou do antebraço.

No caso da tunelização de conduto venoso durante a transposição da veia basílica, sua passagem entre a extremidade proximal e distal é realizada com a utilização da ponta interna, passando o conduto venoso no interior da proteção

Tabela 3 - Desfechos perioperatórios (até 30 dias) - total de 11 pacientes

\begin{tabular}{lc}
\hline Desfechos cirúrgicos & Número de eventos (\%) \\
\hline Hematomas maiores (com necessidade de & $0(0)$ \\
intervenção cirúrgica) & \\
Sangramentos & $0(0)$ \\
Trombose & $0(0)$ \\
Lesões neurológicas & $0(0)$ \\
Insuficiência arterial & $0(0)$ \\
Infecções & $0(0)$ \\
Presença de frêmito no conduto & $11(100)$ \\
\hline
\end{tabular}

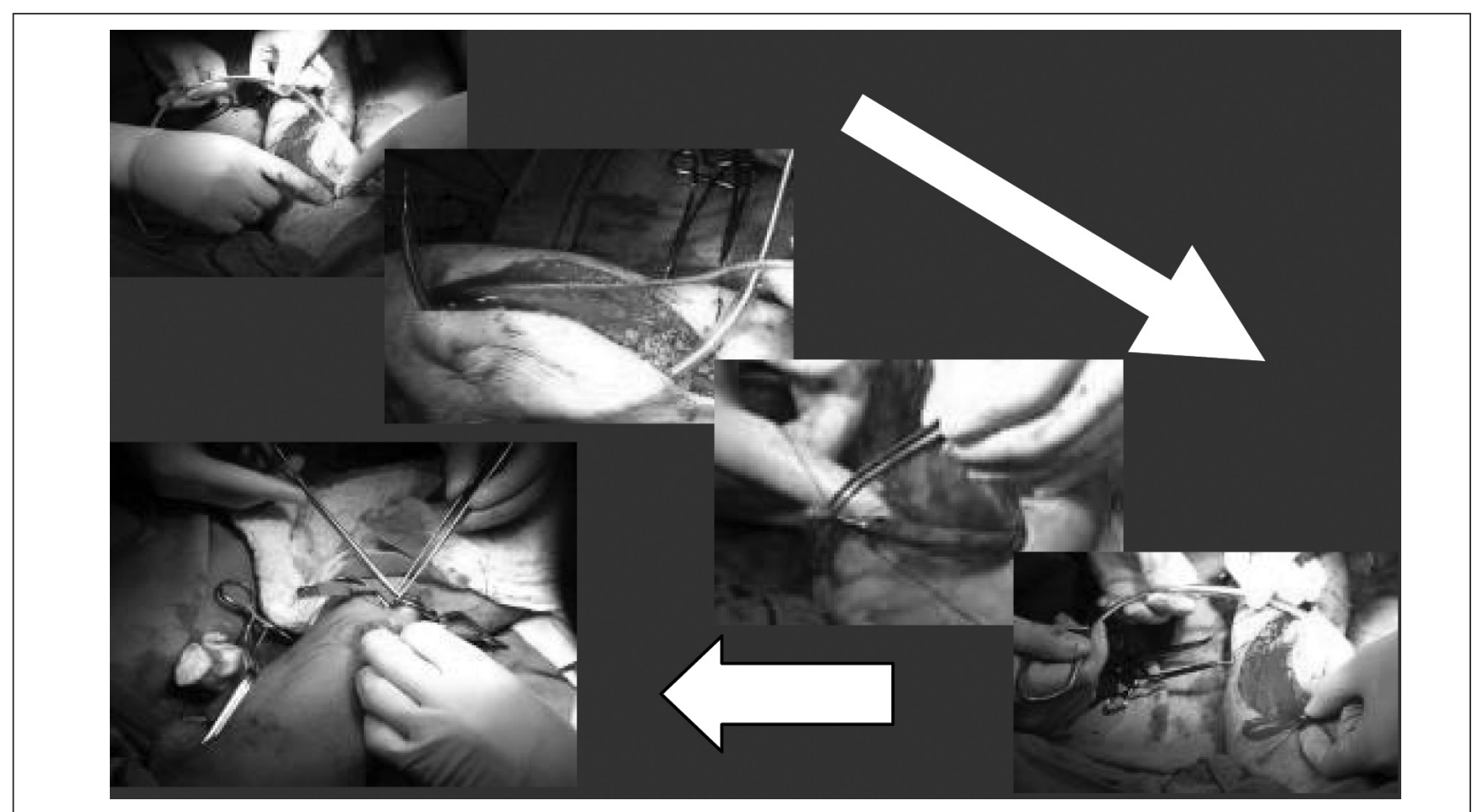

Figura 3 - Tunelização de veia baślica com a proteção da bainha transparente de polietileno. Na segunda imagem, observa-se o tunelizador já posicionado entre os locais escolhidos para a realização da anastomose braquiobasílica. A seguir, ocorre a fixação da veia baślica na haste metálica interna. Na sequência à direita, verifica-se a introdução da veia basílica através da bainha protetora e, à esquerda, a confecção da anastomose braquiobasílica. 
A

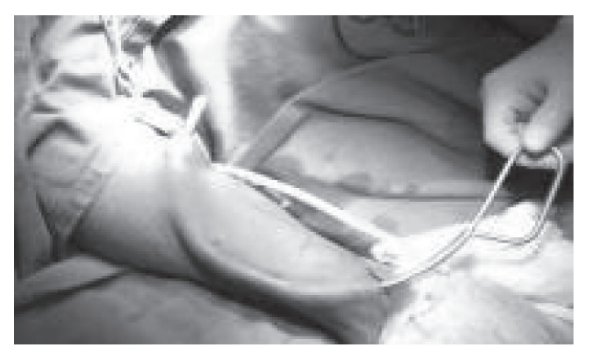

B

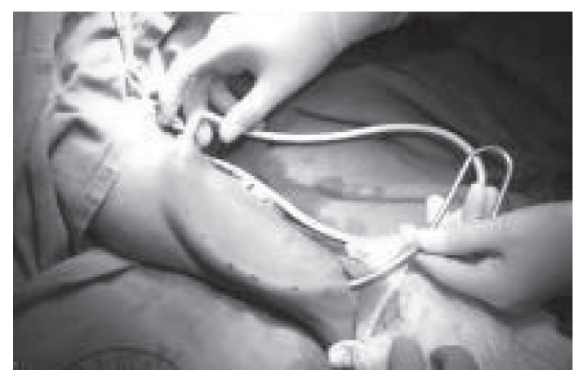

D

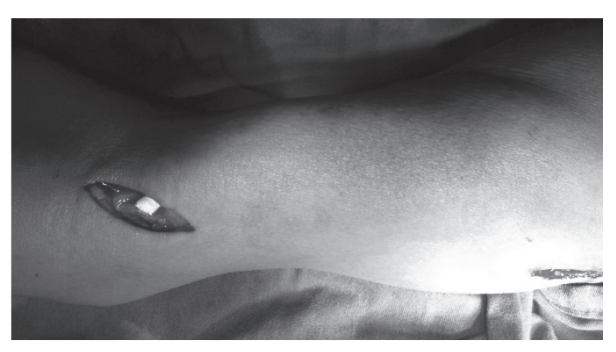

C

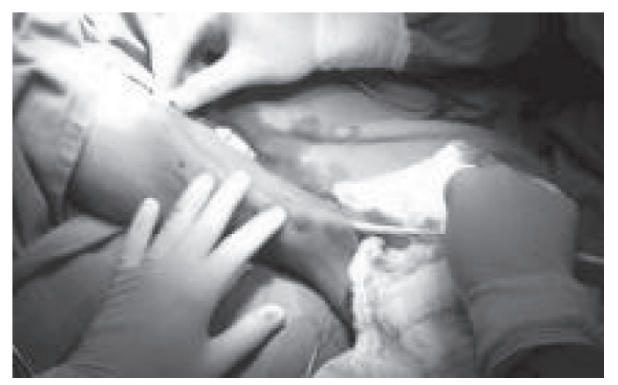

Figura 4 - (A) Confecção do túnel com o passador rígido e curvo, demonstrando a boa adequação do mesmo no tecido celular subcutâneo do braço. (B) Acoplamento com a prótese de politetrafluoroetileno expandido de $6 \mathrm{~mm}$. (C) Sua passagem pelo subcutâneo. (D) Resultado final da tunelização sem incisões escalonadas.

Tabela 4 - Desfechos durante a primeira utilização da fístula arteriovenosa

\begin{tabular}{ll}
\hline Desfechos na primeira diálise & \\
\hline Dificuldades de punção & Número de eventos: 0 \\
Valor do fluxo dialítico & $323 \mathrm{~mL} / \mathrm{min}(295-408 \mathrm{~mL} / \mathrm{min})$ \\
\hline
\end{tabular}

da bainha de polietileno, evitando a torção, estiramento, lesão da ligadura dos ramos e acotovelamentos (Figura 3).

Durante a realização das fístulas axilobraquiais com PTFE, utiliza-se a ponta externa do aparato rígido, permitindo uma acomodação firme com a prótese de diâmetro de $6 \mathrm{~mm}$, assim como uma passagem pela bainha cilíndrica de polietileno, evitando o contato do mesmo com a pele e estruturas adjacentes (Figura 4).

Respeitado o período de maturação de três semanas para prótese sintéticas e de quatro semanas para as transposições de veia basílica, não houve relato de dificuldades técnicas de punção durante a primeira hemodiálise, e o fluxo de diálise médio foi de $323 \mathrm{~mL} / \mathrm{min}$ (Tabela 4).

\section{Discussão}

Quanto maior a perviedade de um acesso vascular, maior a sobrevida dos pacientes com doença renal tratados com hemodiálise ${ }^{3}$. As FAV autólogas representam o padrão ouro para os acessos vasculares nos pacientes dialíticos, pois oferecem uma maior perviedade com um menor risco de complicações a médio e longo prazos, quando comparadas a outros substitutos vasculares com o PTFE ${ }^{4,5}$.

Os enxertos com uso de próteses sintéticas e as transposições venosas são comumente utilizadas na confecção de acessos vasculares, quando as possibilidades de realização de fístulas distais já foram esgotadas ou não existe um conduto venoso adequado. A falha técnica é uma das principais causas de obstrução precoce do enxerto. A torção, a tração, o estiramento, o acotovelamento e o esgarçamento do enxerto podem levar a trombose precoce do mesmo. Além do cuidado no momento da realização das anastomoses, a tunelização é uma etapa importante para o sucesso da cirurgia ${ }^{6-8}$.

A taxa de perviedade primária de uma FAV com prótese sintética é de 60 a $70 \%$ no primeiro ano e de 50 a $60 \%$ nos anos subsequentes, na maioria dos centros ${ }^{9}$. São fatores relacionados quanto à perda da funcionalidade da fístula: sexo feminino, raça negra, idade avançada, tempo de diálise prévio, número de cateteres de diálise anteriores e comorbidades cardiovasculares ${ }^{10}$. Neste estudo, a maioria dos pacientes era do sexo masculino (55\%), raça 
branca (45\%) e apenas $27 \%$ tinham histórico de cardiopatias isquêmicas. Quanto ao uso de cateteres para diálise prévios em veia jugular ou subclávia, 8 (73\%) dos 11 pacientes estudados possuíam 3 ou mais dispositivos antes da confecção da FAV.

A injúria cirúrgica mais comum durante as dissecções abertas para transposições da veia basílica ou para a utilização de próteses na região do braço são as lesões inadvertidas do nervo cutâneo medial do antebraço; por isso, a tunelização do conduto venoso nessa região deve ser feita de forma cuidadosa. A utilização do tunelizador metálico rígido evita dissecções extensas, minimizando as lesões de estruturas vizinhas, além de prevenir lesões estruturais da prótese ou da veia, dando ao conduto uma disposição satisfatória para as punções de diálise $^{11-13}$.

As complicações relacionadas aos AV aumentam com a idade ${ }^{14}$. Grapsa et al., em um estudo incluindo 48 pacientes com mais de 60 anos e 101 pacientes com menos de 60 anos, observaram complicações trombóticas da FAV em torno de $20 \%$ no primeiro grupo e de $13 \%$ no segundo grupo, no período de acompanhamento de 1 ano. Relatou-se taxa de $1,9 \%$ de complicações infecciosas no primeiro grupo. Neste estudo, não se observaram complicações infecciosas, apesar da amostra e tempo de acompanhamento menores ${ }^{15}$.

A confecção de incisões escalonadas para a realização do túnel pode limitar sua adaptação ou até mesmo causar lesões às estruturas, podendo estar envolvida também com complicações infecciosas da ferida operatória e do enxerto ${ }^{2}$. A utilização do aparato metálico rígido e curvo propicia maior proteção, maior facilidade de tunelização e melhor disposição do conduto, sem predispor a infecções relacionadas ao AV neste estudo.

O tempo médio da primeira punção para utilização da fístula foi de 23 dias neste estudo, correspondendo a um tempo menor do que os relatados em outros países, como o Japão e os Estados Unidos, que variam de 25 a 98 dias, respectivamente ${ }^{16}$.

A disposição de sua ponta metálica externa intercambiável permite uma boa adaptação às próteses de PTFE de $6 \mathrm{~mm}$ de diâmetro, assim como ao calibre das veias. Sua bainha cilíndrica externa de polietileno transparente evita o contato do conduto com a pele e estruturas vizinhas, além de permitir visualização direta do conduto durante sua passagem pelo túnel, evitando-se as torções e rotações do mesmo.

Rossi et al. ${ }^{2}$ publicaram sua experiência inicial com um aparato metálico maleável e moldável na tunelização de condutos venosos ou protéticos para enxertos arteriais, obtendo sucesso técnico nos 6 pacientes nos quais utilizouse o tunelizador metálico.

Apesar dos resultados satisfatórios com o uso do tunelizador rígido e curvo na confecção das fístulas de MMSS, a pequena amostra avaliada, assim como o tempo curto de seguimento dos pacientes, limita os resultados deste estudo.

\section{Conclusão}

O tunelizador metálico rígido e curvo pode ser utilizado com segurança, apresentando vantagens nas cirurgias de confecções de acessos venosos para hemodiálise dos MMSS. Suas características de rigidez, concavidade e as características de sua ponta se adéquam bem para a locação dos condutos no subcutâneo durante a confecção das fístulas de braço e antebraço, seja por transposição venosa ou por realização de fístulas com prótese sintéticas.

\section{Referências}

1. Rodríguez-Hernández JA, González-Parra E, Gutiérrez-Julián JM, et al. Guía de acceso vascular en hemodiálisis. Angiología. 2005; 57:117-8.

2. Rossi FH, Izukawa NM, Oliveira LA, et al. Soft moldable universal tunneler in bypass surgery for lower limb ischemia. J Vasc Bras. 2010;9:21-4.

3. Braunwald E, Fauci A, Ajay KS, Barry M. Harrison's principles of internal medicine. $15^{\text {th }}$ ed. Philadelphia McGraw-Hill Medical Publishing Division; 2005:731-40.

4. Nagato Y, Calixto CAN, Brandão ML, et al. Axillary arteriovenous fistula for hemodialysis: case report. J Vasc Bras. 2009;8:371-3.

5. Ekoyang G, Levin NW, Eschbach JW, et al. Continuous quality improvement: DOQI becomes K/DOQI and is updated. National Kidney Foundation's Dialysis Outcome Quality Initiativa. Am J Kidney Dis. 2001; 37:179-94

6. Silva MB Jr, Hobson RW 2nd, Pappas PJ, et al. Vein transposition in the forearm for autogenous hemodialysis access. J Vasc Surg. 1997; 26:981-8.

7. Silva MB Jr, Hobson RW 2nd, Pappas PJ, et al. A strategy for increasing use of autogenous hemodialysis access procedures: impact of preoperative noninvasive evaluation. J Vasc Surg. 1998;27:302-8.

8. Owens CD, Ho KJ, Conte MS. Lower extremity vein graft failure: a translational approach. Vasc Med. 2008;13:63-74.

9. Munda R, First MR, Alexander JW, Linnemann CC Jr, Fidler JP, Kittur D. Polytetrafluoroethylene graft survival in hemodialysis. JAMA. 1983;249:219-22.

10. Wasse H, Speckman RA, Frankenfield DL, Rocco MV, McClellan WM. Predictors of delayed transition from central venous catheter use to permanent vascular access among ESRD patients. Am J Kidney Dis. 2007;49:276-83.

11. Quinn B, Cull DL, Carsten CG. Hemodialysis access: Placement and management of complications. In: Hallet JW Jr, Mills JL, Earnshaw JJ, Reekers JA, Rooke T, editors. Comprehensive vascular and endovascular surgery. London: Mosby Elsevier; 2004. p. 361-90. 
12. Gelabert HA, Freischlag JA. Hemodialysis access. In: Rutherford RB, editor. Vascular surgery. Philadelphia: WB Saunders; 2000. p. 1466-77.

13. Davidson IJ, Cava-Bartsch C. Basilic vein transposition. A case report with contra- lateral removal of a large A-V fistula. J Vasc Access. 2005;6:49-61.

14. Ikizler TA, Himmerlfarb J. Trials and Trade-offs in haemodialysis vascular access monitoring. Nephrol Dial Transplant. 2006;21:3362-3.

15. Grapsa E), Paraskevopoulos AP, Moutafis SP, et al. Complications of vascular access in hemodialysis (HD)--aged vs adult patients. Geriatr Nephrol Urol. 1998:8:21-4.

16. Rayner HC, Pisoni RL, Gillespie BW, Goodkin DA, Akiba T, Akizawa T, Saito A, Young EW, Port FK; Dialysis Outcomes and Practice Patterns Study. Creation, cannulation and survival of arteriovenous fistulae: data from the Dialysis Outcomes and Practice Patterns Study. Kidney Int. 2003;63:323-30.
Correspondência: Heraldo Antonio Barbato Avenida Dr. Dante Pazzanese, 500 - Vila Mariana CEP 04012-180 - São Paulo (SP), Brasil

E-mail:hbarbato@uol.com.br

Contribuições dos autores: Concepção e desenho do estudo: PBM, HAB Análise e interpretação dos dados: PBM, HAB Coleta de dados: PBM, FMRA, BLA, ESJ, BUS Redação do artigo: PBM, BUS Revisão crítica do artigo: $H A B, N M I$ Aprovação final do artigo*: PBM, HAB, FMRA, FHR, BLA, ESJ, NMI, BUS Análise estatística: PBM, FMRA Responsabilidade geral do estudo: PBM, HAB, NMI Informações sobre financiamento: N/A *Todos os autores leram e aprovam a versão final submetida ao J Vasc Bras. 